\title{
Mechanisms of Colorectal Cancer Cell Growth and Metastasis Inhibition By Carp-1 Functional Mimetic-4
}

\author{
Abdelkader E. Ashour ${ }^{1}$, Anwar A. Almuslim², Ashok Kumar ${ }^{3}$, Khairy M. A. Zoheir ${ }^{4}$, Rehan \\ Ahmed $^{5}$, Sheikh F. Ahmad ${ }^{2}$, Sabry M. Attia ${ }^{2,5}$, Khalid M. AlGhamdi ${ }^{3}$, Adel R. A. Abd-Allah ${ }^{6}$ \\ ${ }^{1}$ Deppartment of Basic Medical Sciences, Kulliyyah of Medicine, International Islamic \\ University Malaysia \\ ${ }^{2}$ Department of Pharmacology and Toxicology, College of Pharmacy, King Saud University, \\ Riyadh, Saudi Arabia \\ ${ }^{3}$ Vitiligo Research Chair, College of Medicine, King Saud University, Riyadh, Saudi Arabia \\ ${ }^{4}$ Cell Biology Department, National Research Centre, Cairo, Egypt \\ ${ }^{5}$ Colorectal Research Chair, Department of Surgery, King Khalid University Hospital, King \\ Saud University \\ ${ }^{6}$ Department of Pharmacology and Toxicology, Faculty of Pharmacy, Al-Azhar University, \\ Cairo, Egypt.
}

Presenter: Abdelkader Elbadawy Ashour

Introduction: Colorectal cancer (CRC) constitutes one of the most aggressive malignancies worldwide and in Malaysia. Due to high recurrence rate and toxic side effects associated with radiation and chemotherapies, new agents are urgently needed. CARP-1 is a peri-nuclear phospho-protein which plays a dynamic role in regulating cell growth and apoptosis. CARP-1 functional mimetics (CFMs) are a class of compounds that stimulate CARP-1. CFM-4, a lead compound, was shown to suppress growth and metastasis of various cancers, other than CRC. We hypothesized that CFM-4 inhibits proliferation and metastasis in CRC. Materials and method: CFM-4 anti-cancer effects of on CRC cells were investigated using MTT assay, Annexin V/Propidium iodide (PI) apoptosis assay, cell cycle analysis, quantitative real-time PCR (qRT-PCR) and Western blotting. Antimetastatic activities were assessed by migration, colony formation and invasion assays. Results: CFM-4 inhibited CRC cell proliferation and was much more potent than the classical anti-CRC 5-fluorouracil. These effects were shown to be mediated at least in part by stimulating apoptosis, as indicated in our Annexin V/PI assay results. Cell cycle analysis showed that CFM-4 induced G2/M phase arrest. Molecularly, qRT-PCR results revealed that CFM-4 promoted intrinsic apoptosis by upregulating expression of caspase-8 and -9 , p53, PUMA and Noxa, and stimulated extrinsic apoptosis by enhancing expression of death receptors (DR4 and DR5). CFM-4 upregulated NF- $\mathrm{k}$ B signaling inhibitor A20-binding inhibitor protein and the PI3K negative regulator PTEN. Western blot analysis results revealed that CFM-4 enhanced expression of CARP. 1 , caspase- 8 and executioner caspase-3. Metastatic properties of the CRC cells were reduced by $\mathrm{CFM}-4$ through blocking their capabilities to form colonies, migrate and invade through the matrix-coated membranes. Conclusion: The potent antitumor and anti-metastatic properties of CFM-4 against CRC are due to collective pro-apoptotic, anti-proliferative and anti-metastatic activities. Together our data warrants further investigations of CFM-4 as potential anti-tumor agent for CRC malignancy and metastasis. 